\title{
Treatment response in major depression: effects of personality dysfunction and prior depression
}

\author{
P. Gorwood, F. Rouillon, C. Even, B. Falissard, E. Corruble and P. Moran
}

\section{Background}

The impact of personality dysfunction on the outcome of treatment for depression remains debated.

\section{Aims \\ To examine the relationship between the number of prior depressive episodes, personality dysfunction and treatment response for depression.}

\section{Method}

In a large sample $(n=8229)$ of adult out-patients with a major depressive episode (DSM-IV), personality dysfunction was assessed using the Standardised Assessment of Personality Abbreviated Scale (SAPAS). Potential predictors of treatment response at 6 weeks were examined via structural equation modelling.

\section{Results}

The amount of personality dysfunction and number of prior episodes of depression were both associated with poor response to treatment. Once personality dysfunction was controlled for, the number of prior episodes of depression was not associated with treatment response.

\section{Conclusions}

Personality dysfunction is associated with impaired shortterm response to antidepressant treatment in major depression. The apparent detrimental effect of prior depression on treatment response may be accounted for by pre-existing personality dysfunction.

\section{Declaration of interest}

P.G. has received grants from Eli Lilly and Servier, honoraria from Bristol-Myers Squibb, Lundbeck, Servier and UCB-Pharma and has participated in advisory boards for Janssen, Servier and Wyeth.
The impact of comorbid personality dysfunction on the outcome of treatment for depression remains unclear. Some systematic reviews have pointed towards there being no association, ${ }^{1,2}$ whereas others have reported as much as a twofold increase in poor response among individuals with depression with comorbid personality dysfunction. ${ }^{3}$ Equally, the nature of the relationship between prior depression and personality dysfunction is unclear. Personality dysfunction may emerge as a complication or 'scar' following repeated depressive episodes; indeed currently this is formally recognised in ICD-10, under the category 'enduring personality change after psychiatric illness' (F62.1). ${ }^{4}$ Nonetheless, empirical support for the category is limited and it remains possible that personality dysfunction may have a pathoplastic effect on the manifestation and course of subsequent depressive episodes. With these issues in mind, we set out to examine the relationship between the number of prior depressive episodes, risk of personality dysfunction and treatment response for current depression in a large out-patient cohort.

\section{Method}

The rationale and full details of the methods used in the study are described elsewhere. ${ }^{5}$ In brief, the original sample was recruited to a previous study devoted to memory impairments in people with major depressive dysfunction. In total 1844 medical doctors included at least 1 depressed patient in the study. Inclusion criteria were as follows: meeting DSM-IV ${ }^{6}$ criteria for a major depressive disorder; people for whom a new (or different) prescription of antidepressant had to be made (all antidepressants were accepted in order to reflect usual clinical practice) for a major depressive episode (average length 8.4 weeks, s.d. $=10.8$ ); over 18 years old, speaking fluent French, with social security coverage and able to give informed consent. Exclusion criteria were the diagnosis of bipolar affective disorder and the use of a mood stabiliser in treatment. The DSM-IV criteria for a major depressive episode were assessed by the clinician. The study was approved by the local research ethics committee and all participants gave full written informed consent. A total of 9515 people were included in the study and all were followed up at 6 weeks.

\section{Instruments}

The Hospital Anxiety and Depression Scale $(\mathrm{HAD})^{7}$ was chosen as a self-report instrument to measure symptom severity and was completed by all participants at the first and the second visit. A score above eight for the depression domain and the presence of five symptoms or more, i.e. the DSM-IV diagnosis of major depressive disorder, were required for inclusion. Responders were defined as having at least a $50 \%$ decrease in their depression score between the two visits.

The initial assessment also included the number of past depressive episodes, either treated or not treated with an antidepressant, and the cumulative length of past mood disorder.

A brief screening interview for personality disorders (Standardised Assessment of Personality - Abbreviated Scale, SAPAS $)^{8}$ was also administered to all participants $(n=9515)$. The SAPAS is a short, simple-to-administer screening test, which demonstrates good psychometric properties. ${ }^{8,9}$ The interview consists of eight questions, corresponding to a descriptive statement about the person. Each of the questions can be scored 0 (no) or 1 (yes) and the scores on the eight items are added together to produce a total score of between 0 and 8 .

\section{Statistics}

Variables, including SAPAS total score, were graphically checked for normality of distribution (quantile-quantile plots) before using parametric statistics.

Parametric correlation (Pearson test) was used to compare two continuous variables, and analysis of variance (ANOVA) for the 
analysis of the role of a qualitative factor to explain continuous parameters.

Structural equation modelling is a statistical technique for estimating so-called causal relationships using a combination of qualitative causal assumptions. The structural equation modelling was performed, using Mplus software 10 (www.statmodel.com), to analyse the impact of adding the SAPAS score as an intermediate step between the number of past depressive episodes and treatment response, while tackling the impact of confounding factors. In these models, the SAPAS score was considered as an ordinal variable.

\section{Results}

The final sample for analysis consisted of 8229 people $(86.50 \%$ of the initial sample). Individuals were excluded at baseline if the HAD score was below eight for depression and/or anxiety (644 people) or because of missing or incomplete data $(n=642)$, representing a $13.59 \%$ rate of protocol violation and losses to follow-up. The subsample of the latter individuals had shorter length of the present episode $(t=2.15$, d.f. $=6798, P=0.016)$, were more frequently males $\left(\chi^{2}=172.9\right.$, d.f. $\left.=8, P<0.001\right)$ and had a lower final HAD score for depression $(t=3.974$, d.f. $=9512$, $P<0.001)$ and for anxiety $(t=5.212$, d.f. $=9512, P<0.001)$ and accordingly included a higher proportion of responders $\left(\chi^{2}=3.71\right.$, d.f. $\left.=1, P=0.012\right)$.

At the first visit, the mean SAPAS score was 3.82 (s.d.=1.82). After 6 weeks of antidepressant treatment, 2424 participants
(29.5\%) were responders according to the HAD score at the second visit. Their characteristics are displayed in Table 1 .

Responders were more likely to be male, younger, single or married (rather than divorced or widowed), of a higher educational level and engaged in professionally active work at baseline. Furthermore, they were depressed for a shorter period of time, regarding the present episode but also the cumulative length of depressive disorder, and, accordingly, had less past depressive episodes (Table 1). Treatment response was indeed associated with a lower number of previous depressive episodes (odds ratio $(\mathrm{OR})=0.931,95 \%$ CI $0.897-0.966, P<0.001$ ). In addition, there was a strong statistical association between the SAPAS score and treatment response, the former being higher in the non-responder group $(t=4.92$, d.f. $=8227, P<0.001)$.

The relationships between the SAPAS score and sociodemographic and clinical variables are displayed in Table 2.

The SAPAS score was significantly correlated with all clinical parameters and all sociodemographic factors, except age and gender. The variable most highly correlated $(r=0.148, P<0.001)$ with SAPAS score was the number of past episodes of depression, and there was a relatively linear association between the two variables (Fig.1).

In order to examine more closely the association between comorbid personality dysfunction and treatment response, we used structural equation modelling. As anticipated, the number of past episodes was robustly associated with treatment response $(P<0.001)$. This association was still observed after controlling for the period of time between the initial and the follow-up visit (which was fixed at 6 weeks, but in fact had some variance),

\begin{tabular}{|c|c|c|c|}
\hline Characteristics & $\begin{array}{c}\text { Non-responders } \\
\quad(n=5805)\end{array}$ & $\begin{array}{l}\text { Responders } \\
(n=2424)\end{array}$ & $P$ \\
\hline Gender, female: \% & 71.25 & 68.19 & $<0.001$ \\
\hline Age, years: mean (s.d.) & $48.94(14.37)$ & $45.80(13.11)$ & $<0.001$ \\
\hline Marital status (\%) & & & $<0.001$ \\
\hline Single & 17.08 & 19.22 & \\
\hline Married & 55.24 & 57.34 & \\
\hline Divorced & 18.33 & 17.14 & \\
\hline Widow & 9.34 & 6.30 & \\
\hline Educational level (\%) & & & $<0.001$ \\
\hline School & 51.15 & 44.29 & \\
\hline College & 29.51 & 31.21 & \\
\hline University & 19.34 & 24.50 & $<0.001$ \\
\hline \multicolumn{4}{|l|}{ Professional activity (\%) } \\
\hline Currently active & 55.49 & 63.57 & \\
\hline Unemployed & 12.44 & 12.43 & \\
\hline Retired & 20.90 & 14.76 & \\
\hline Student & 0.07 & 0.21 & \\
\hline Other & 11.10 & 9.03 & \\
\hline $\begin{array}{l}\text { Number of past episodes, mean (s.d.) } \\
\text { Episodes, \% }\end{array}$ & $1.00(1.48)$ & $0.87(1.26)$ & $<0.001$ \\
\hline 0 episodes & 48.89 & 53.09 & \\
\hline 1 episode & 26.15 & 24.27 & \\
\hline 2 episodes & 14.57 & 14.37 & \\
\hline 3 episode & 6.48 & 5.26 & \\
\hline 4 episode & 2.40 & 2.21 & \\
\hline 5 or more episodes & 2.62 & 1.70 & \\
\hline Length of current episode, weeks: mean (s.d.) & $8.92(12.14)$ & $7.09(6.80)$ & $<0.001$ \\
\hline Length of depressive disorder, weeks: mean (s.d.) & $23.00(37.74)$ & $17.16(22.27)$ & $<0.001$ \\
\hline Average SAPAS score, mean (s.d.) & $3.89(1.78)$ & $3.67(1.91)$ & $<0.001$ \\
\hline Baseline Hospital Anxiety and Depression Scale score, mean (s.d.) & $28.11(5.12)$ & $30.23(4.98)$ & $<0.001$ \\
\hline Number of DSM-IV criteria for major depressive disorder, mean (s.d.) & $6.56(1.29)$ & $6.75(1.16)$ & $<0.001$ \\
\hline Delays between the two visits, days: mean (s.d.) & $41.90(9.22)$ & $42.56(8.17)$ & 0.001 \\
\hline
\end{tabular}




Table 2 Relationships between the level of personality dysfunction (according to the Standardised Assessment of Personality -
Abbreviated Scale score) and different clinical parameters in a sample of 8229 depressed out-patients
Variable

educational status, marital status, gender, professional activity and age (Fig. 2a, $P=0.02$ ). When the SAPAS score was then added to the equation as a mediating variable (Fig. $2 \mathrm{~b}$ ) a highly significant association was detected between SAPAS score and treatment response and with the number of past depressive episodes $(P<0.001$ for both associations). However, adding this path diminished the role of past depressive episodes below the $5 \%$ level of significance $(P=0.21)$.

\section{Discussion}

\section{Main findings}

In a sample of more than 8000 out-patients treated for a major depressive episode, the level of personality dysfunction and the number of previous depressive episodes were both associated with poor short-term treatment response. In addition, a linear association was detected between the number of previous episodes of depression and the severity of personality dysfunction. Finally, in a structural equation model examining predictors of treatment response, the number of previous episodes of depression had little impact on treatment outcome, once the effect of other factors, including personality dysfunction, had been controlled for.

\section{Explanations for the detected associations}

The two potential but not mutually exclusive explanations for this association are referred to as the 'scar' and the 'vulnerability' hypotheses. The vulnerability hypothesis states that people with

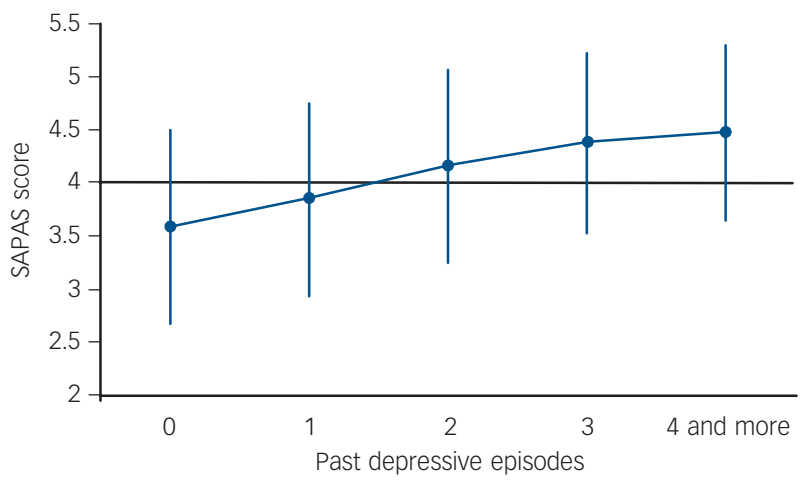

Fig. 1 Level of initial Standardised Assessment of Personality - Abbreviated Scale (SAPAS) score according to the number of past depressive episodes in a sample of 8229 depressed out-patients. higher levels of personality dysfunction are at increased risk of recurrence of depression ${ }^{10}$ whereas the alternative scar hypothesis claims that cumulative episodes of depression have a detrimental effect on personality that would secondarily impair treatment response. The 'scarring' effect of cumulative depressive episodes on personality has been previously explored. In a prospective study that assessed a sample of 1733 twins from female-female pairs, Kendler et al found that neuroticism was increased after a major depressive episode. ${ }^{11}$ Increasing vulnerability through new episodes is also in accordance with the kindling hypothesis proposed by Post. ${ }^{12}$ Conversely, in a 6 -year follow-up study of 812 first-degree relatives of people with depression, Shea et al, observed that personality status was relatively unchanged after, compared with before, the episode. ${ }^{13}$ We were unable to distinguish between these two alternative explanations as

(a)

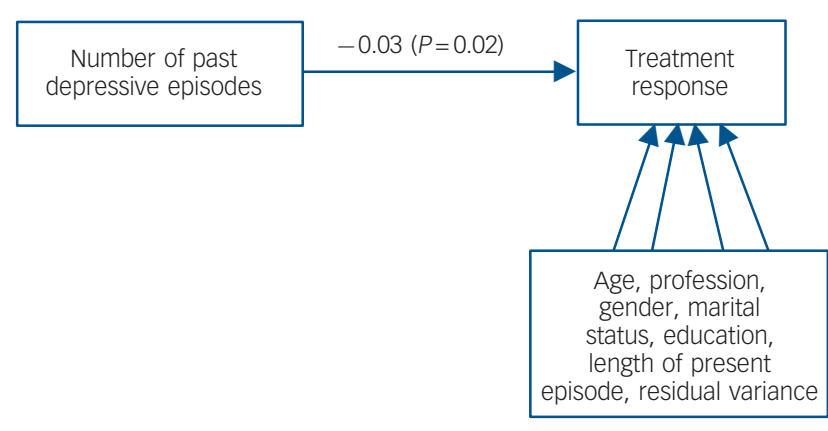

(b)

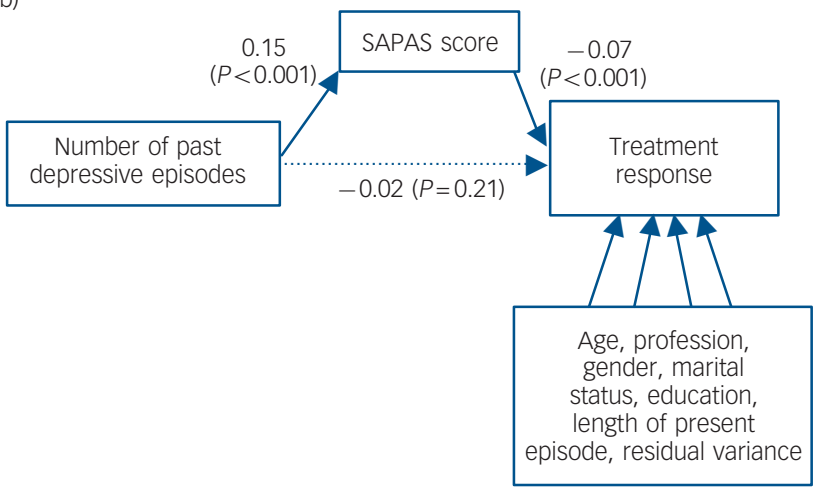

Fig. 2 Structural equation model accounting for treatment response in 8229 depressed out-patients, (a) without taking into account the Standardised Assessment of Personality Abbreviated Scale (SAPAS) score, (b) including the SAPAS score. 
personality dysfunction and prior depression were assessed concurrently in our study. Nevertheless, our results from structural equation modelling suggest that the effect of past depressive episodes on treatment response may be accounted for by the level of pre-existing personality dysfunction.

\section{Methodological considerations}

To the best of our knowledge, this is the largest study of depression and personality dysfunction to date. We adopted rigorous case definition for depression, a prospective follow-up, and appropriate statistical methods. Nevertheless, there were some limitations.

First, we observed a $29.5 \%$ treatment response rate, which may appear low compared with what is generally reported in controlled clinical trials. However, in the STAR ${ }^{\star} \mathrm{D}$ trial (Sequenced Treatment Alternatives to Relieve Depression), ${ }^{14}$ the participants who were engaged in the second treatment step experienced a response rate of $28.5 \%$ after 12 to 14 weeks, very close to the proportion of responders we recorded at 6 weeks.

Second, we deliberately chose to use a brief measure of personality dysfunction, in order to facilitate efficient fieldwork with a large sample in a real-life clinical setting. Arguably, a more detailed measure of personality dysfunction may have been preferable. However, against this, the SAPAS demonstrates good psychometric properties ${ }^{8}$ and is a valid screen for personality disorder.

Third, the SAPAS instrument was designed to screen for personality disorder in a psychiatric setting and we do not know its predictive value in primary care. Furthermore, we restricted the analyses to the global SAPAS score and it is unclear whether the SAPAS is a truly continuous variable in relation to personality. Nevertheless, in a large unrelated study, global SAPAS score has been shown to independently predict retention in treatment for people with personality disorder, over a 30 -month period and the global score therefore appears to have predictive validity and thus clinical utility. ${ }^{15}$

Fourth, we assessed personality dysfunction and prior depression concurrently. Acute depression can colour the assessment of personality and hence the personality dysfunction detected in this study could represent a manifestation of depression, ${ }^{16}$ although self-reports are considered by some authors as concurrently reliable and valid during acute depressive episodes. ${ }^{16}$

Fifth, the effect size explaining treatment response in this study is weak, which may reduce the utility of the present results at the individual level. Indeed, the standardised regression coefficient between the number of past episodes and treatment response, when all confounders are being controlled for, is of smal amplitude $(r=-0.03)$. Nevertheless, numerous factors are known to be involved in treatment response, and were also detected in our sample (Table 1). Controlling for such a larger number of important variables (with the structural equation modelling) is logically reducing the regression coefficient. Independent replication of the present finding would be the most appropriate approach to eliminate a chance finding.

Finally, we did not measure a number of potential confounding factors, including comorbid addiction - such factors would be important to capture in any future attempts to replicate our findings.

The relative 'vulnerability' and 'scarring' effects on treatment response were difficult to disentangle in this study and further research should investigate this issue within prospective studies with multiple assessment periods. From a clinical perspective, comorbid personality dysfunction should not be regarded as an inescapably poor prognostic indicator. However, our findings emphasise the challenging requirement of treating both personality dysfunction and depression, and hence the potential merits of combining complex psychotherapeutic interventions with pharmacological treatments for depression.

\section{P. Gorwood, MD, PhD, F. Rouillon, MD, PhD, C. Even, MD, PhD, INSERM U675, Centre de Psychiatrie et Neurosciences and Paris-Descartes University and Sainte- Anne Hospital (CMME), Paris, France; B. Falissard, MD, PhD, INSERM U669 and University Paris-Sud and Paris Descartes, Paris, France; E. Corruble, MD, PhD INSERM U669 and Paris Sud University, AP-HP (Assistance. Publique, Hôp, PhD, Paris), Department of Psychiatry, Bicêtre University Hospital, Le Kremlin Bicêtre, France, P. Moran, MD, Health Services and Population Research Department, Institute of Psychiatry, King's College London, London, UK}

Correspondence: Philip Gorwood, MD, PhD, INSERM U675,

CMME-Sainte-Anne Hospital, 100 rue de la Santé, 75014 Paris, France.

Email : p.gorwood@ch-sainte-anne.fr

First received 9 Apr 2009, final revision 11 Oct 2009, accepted 21 Oct 2009

\section{Funding}

The present work was supported by a research grant from Servier.

\section{References}

1 Mulder RT. Personality pathology and treatment outcome in major depression: a review. Am J Psychiatry 2002; 159: 359-71.

2 Kool S, Schoevers R, de Maat S, Van R, Molenaar P, Vink A, et al. Efficacy of pharmacotherapy in depressed patients with and without personality disorders: a systematic review and meta-analysis. J Affect Disord 2005; 88 269-78.

3 Newton-Howes G, Tyrer $P$, Johnson T. Personality disorder and the outcome of depression: meta-analysis of published studies. Br J Psychiatry 2006; 188: 13-20.

4 World Health Organization. The ICD-10 Classification of Mental and Behavioural Disorders: Clinical Descriptions and Diagnostic Guidelines. WHO, 1992.

5 Gorwood P, Corruble E, Falissard B, Goodwin G. Toxic effects of depression on brain function: impairment of delayed recall and the cumulative length of depressive disorder in a large sample of depressed outpatients. Am J Psychiatry 2008; 165: 731-9.

6 American Psychiatric Association. Diagnostic and Statistical Manual of Mental Disorder (4th edn) (DSM-IV). APA, 1994.

7 Zigmond AS, Snaith RP. The Hospital Anxiety and Depression Scale. Acta Psychiatr Scand 1983; 67: 361-70.

8 Moran P, Leese M, Lee T, Walters P, Thornicroft G, Mann A. Standardised Assessment of Personality - Abbreviated Scale (SAPAS): preliminary validation of a brief screen for personality disorder. Br J Psychiatry 2003; 183: $228-32$.

9 Hesse M, Rasmussen J, Pedersen MK. Standardised assessment of personality - a study of validity and reliability in substance abusers. BMC Psychiatry 2008; 8: 7.

10 Johnson JG, Cohen P, Kasen S, Ehrensaft MK, Crawford TN. Personality disorder traits associated with risk for unipolar depression during middle adulthood. Psychiatry Res 2005; 136: 113-21.

11 Kendler KS, Neale MC, Kessler RC, Heath AC, Eaves LJ. A longitudinal twin study of personality and major depression in women. Arch Gen Psychiatry 1993; 50: 853-62.

12 Post RM. Kindling and sensitization as models for affective episode recurrence, cyclicity, and tolerance phenomena. Neurosci Biobehav Rev 2007; 31: 858-73.

13 Shea MT, Leon AC, Mueller TI, Solomon DA, Warshaw MG, Keller MB. Does major depression result in lasting personality change? Am J Psychiatry 1996; 153: $1404-10$

14 Rush AJ, Trivedi MH, Wisniewski SR, Nierenberg AA, Stewart JW, Warden D, et al. Acute and longer-term outcomes in depressed outpatients requiring one or several treatment steps: a STAR*D report. Am J Psychiatry 2006; 163: 1905-17.

15 Crawford MJ, Price K, Gordon F, Josson M, Taylor B, Bateman A, et al. Engagement and retention in specialist services for people with personality disorder. Acta Psychiatr Scand 2009; 119: 304-11.

16 Costa PT Jr, Bagby RM, Herbst JH, McCrae RR. Personality self-reports are concurrently reliable and valid during acute depressive episodes. I Affect Disord 2005; 89: 45-55. 